\title{
GLARE TESTING IN PSEUDOPHAKES WITH POSTERIOR CAPSULE OPACIFICATION
}

\author{
PALANISWAMY SUNDERRAJ, JOSE R. VILLADA, PATRICK W. JOYCE, ALLAN WATSON \\ Southport
}

\begin{abstract}
SUMMARY
We measured Snellen visual acuity under standard and glare conditions using the Allergan Humphrey 570 autorefractor in 46 pseudophakes (46 eyes) undergoing Neodymium-YAG laser posterior capsulotomy before and after treatment. All the patients studied had a best corrected vision of at least $6 / 12$ but complained of visual disability and had clinically observable posterior capsule opacification. The patients did not have other known causes of glare disability. Following posterior capsulotomy, $30(65 \%)$ patients showed an improvement in standard Snellen visual acuity and $44(97 \%)$ an improvement in Snellen visual acuity under glare conditions. This difference was statistically significant $(p<0.001)$. Of the 16 patients whose standard Snellen visual acuity did not improve after posterior capsulotomy, 14 showed a reduction in glare disability. Glare testing was clinically useful in evaluating pseudophakes with posterior capsule opacification who complained of visual disability in spite of good Snellen visual acuity under standard testing conditions.
\end{abstract}

Glare may be defined as the reduction in visual performance associated with intraocular scattering of light. Glare symptoms may be induced by an incomplete opacification of the ocular media such as corneal scars, cataract, ${ }^{1}$ and posterior capsule opacification following extracapsular cataract extraction (ECCE). ${ }^{2}$ In patients with opacified posterior capsules, improved visual acuity and reduced glare disability are usually achieved by performing surgical or laser posterior capsulotomy.,

Traditional high contrast Snellen visual acuity testing provides incomplete information about the patients' ability to function in the real, multi-contrast world outside the examination room. Glare testing complements this information by objectively documenting the debilitating effect of light scatter from media opacity. This mode of vision testing may be clinically useful in evaluating patients with cataracts $^{1}$ and pseudophakes with posterior capsule opac-

Correspondence to: Mr P. Sunderraj, Medical Adviser, CIBA Vision, Hedge End, Southampton SO3 3LG. ification ${ }^{4}$ who have good Snellen visual acuity but complain of visual diability. A previous study of glare testing in posterior capsule opacification ${ }^{4}$ included a large proportion of patients with poor Snellen visual acuity. This study measured visual acuity under standard and glare conditions before and after treatment, in patients with good Snellen visual acuity who underwent Neodymium-YAG laser posterior capsulotomy.

\section{PATIENTS AND METHODS}

\section{Instrument}

The Allergan-Humphrey 570 autorefractor is a table-top instrument which has the capacity to provide objective autorefraction values. These values can be subjectively verified using built-in lenses and Snellen acuity targets. The standard Snellen acuity target has a $100 \%$ contrast of the letters relative to the background with a source luminance of $160 \mathrm{~cd} / \mathrm{m}^{2}$ or $47 \mathrm{fL}$. The effect of glare can be tested by employing a low contrast (13\%) Snellen acuity target and two bars of light, one above and one below the acuity letters. The source luminance is $18,400 \mathrm{~cd} / \mathrm{m}^{2}$ or $5500 \mathrm{fL}$ with an illuminance of $36.8 \mathrm{lux}\left(1 \mathrm{~m} / \mathrm{m}^{2}\right)$ at the cornea from both glare sources.

\section{Patients}

We prospectively studied all patients with posterior chamber IOLs who underwent Neodymium-YAG laser posterior capsulotomy between May 1990 and March 1991. The indication for laser capsulotomy in these patients was a complaint of visual disability in the presence of clinically observable posterior capsule opacification. For the purposes of this study we included eyes with a best corrected vision of at least $6 / 12$ and only one eye per patient. We excluded eyes with corneal opacities, pupillary abnormalities, decentred implants, multifocal implants, vitreous opacities, degenerative changes in the retina or macula, glaucoma and other optic nerve disease and other known causes of glare disability.

\section{Methods}

Pre-operative measurements were performed with the 


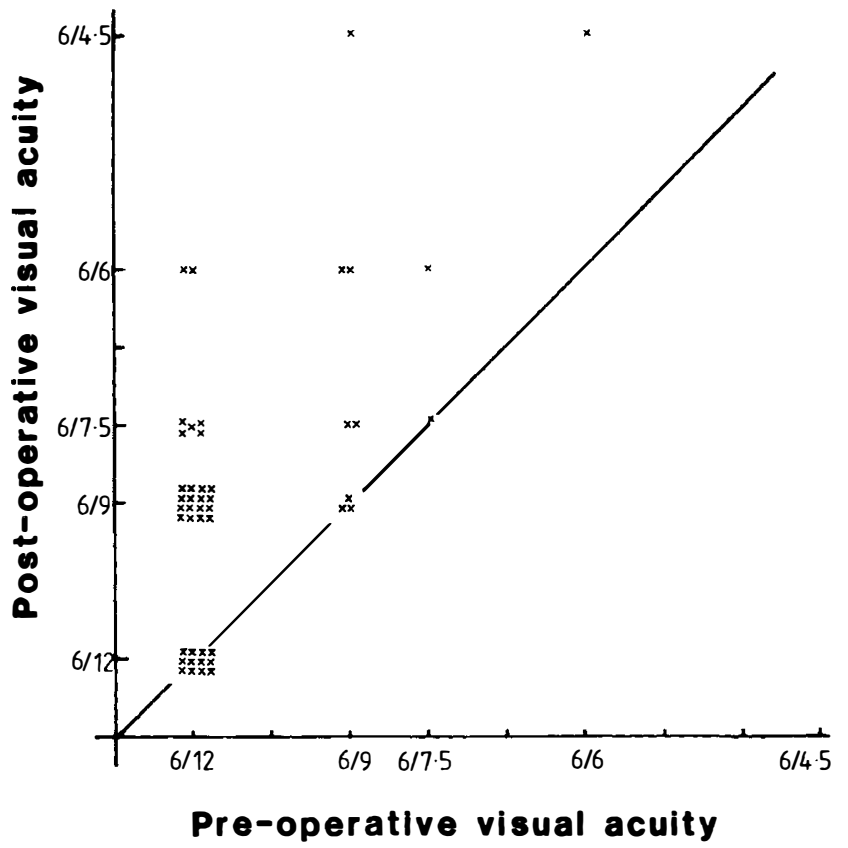

Fig. 1. Comparison of pre-operative and post-operative Snellen visual acuity under standard testing conditions.

Allergan Humphrey 570 autorefractor in a darkened room up to a week preceding the capsulotomy. The standard Snellen visual acuity of the study eye was recorded using the $100 \%$ contrast Snellen acuity target. Objective auto-

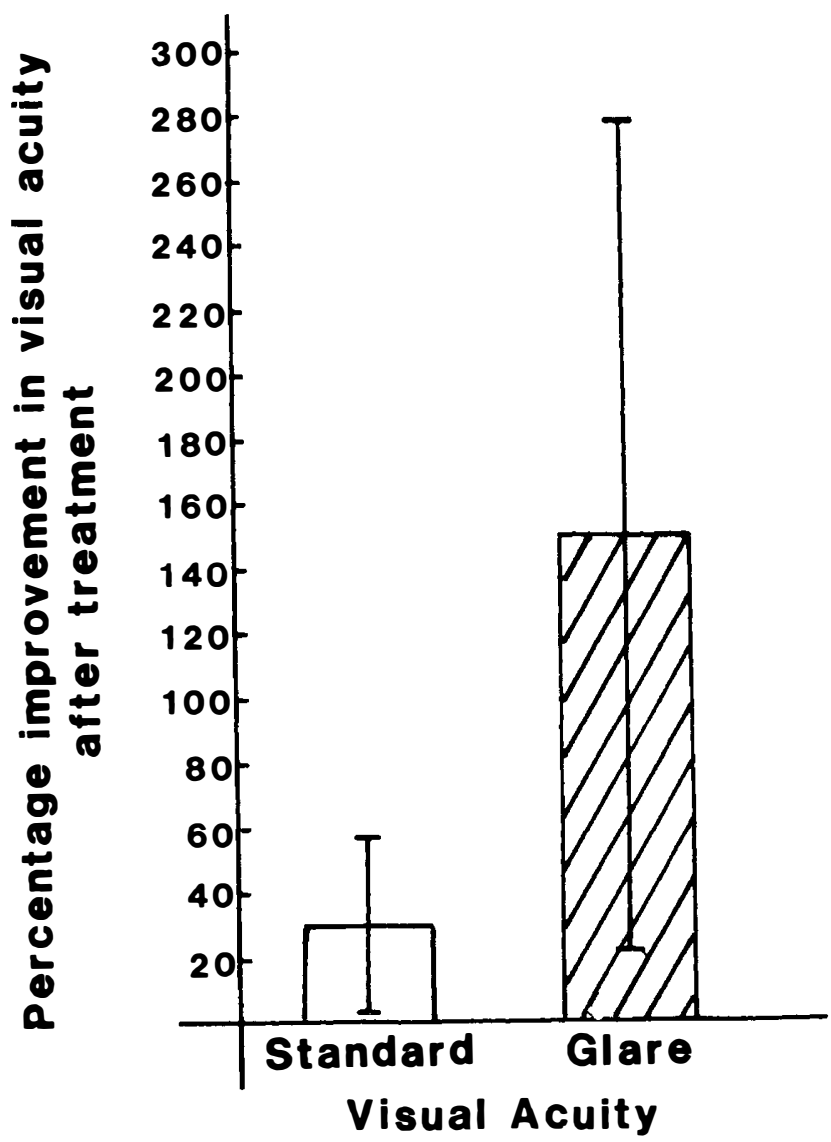

Fig. 2. Percentage improvement in mean Snellen visual acuity under standard and glare conditions following posterior capsulotomy. refraction values were then obtained and subjectively verified using the built-in lenses to give the best-corrected standard Snellen visual acuity. With the corrective lenses in place, the patients were offered the low contrast (13\%) Snellen acuity target. When they were used to this new target, the glare sources in the AH 570 autorefractor were switched on and the maximal Snellen visual acuity obtained under glare conditions was recorded.

All posterior capsulotomies were performed in a single treatment session with a Q-switched Neodymium-YAG laser. In all cases, a good gap was created in the visual axis. Measurements of Snellen visual acuity under standard and glare conditions, as already described, were done between three and four weeks after the capsulotomy without knowledge of the pre-operative data. All the patients were tested by the same operator (PSR). All Snellen visual acuities recorded refer to best-corrected visual acuities under similar conditions.

\section{Statistical analysis}

The paired ' $t$ ' test was used to assess the statistical significance of the improvement in mean Snellen visual acuities under standard and glare conditions before and after posterior capsulotomy.

The Chi-squared $\left(\mathrm{X}^{2}\right)$ test was used to assess the statistical significance of the difference between Snellen visual acuity under standard conditions and Snellen visual acuity under glare conditions in the number of patients showing an improvement following posterior capsulotomy.

\section{RESULTS}

A total of 48 patients (48 eyes) who fulfilled the inclusion and exclusion criteria were studied. Of these, two patients did not return for post-operative measurements. in the pre-

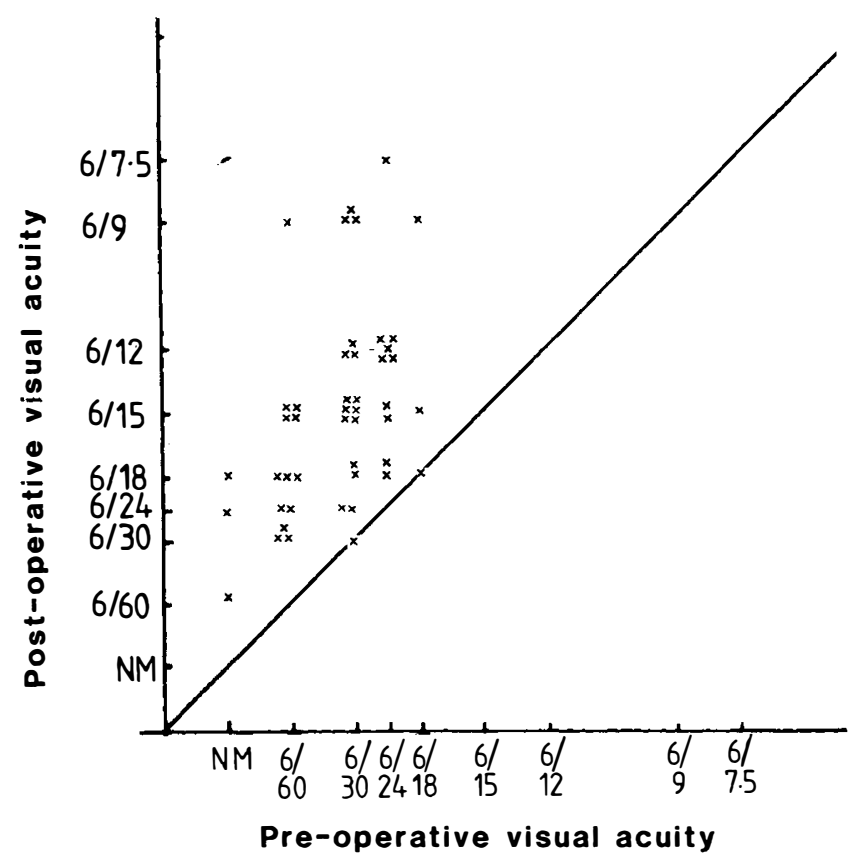

Fig. 3. Comparison of pre-operative and post-operative Snellen visual acuity under glare conditions. (Footnote: NM-not measureable). 
scribed time period. The results of the remaining 46 patients were analysed. There were 28 women and 18 men with a mean age of 71 (SD 8) years.

Posterior capsulotomy produced an improvement in standard Snellen visual acuity in 30 65\%) patients (Fig. 1.). Following laser capsulotomy there was a statistically significant $(\mathrm{p}<0.001)$ improvement in mean standard Snellen visual acuity from 0.56 (SD 0.11 ) to 0.71 (SD 0.19 ), an increase of 30 (SD 27)\% (Fig. 2.). A mean visual acuity of 0.25 equates with $6 / 24,0.5$ with $6 / 12,0.8$ with $6 / 7.5$ and so on.

Snellen visual acuity under glare conditions improved in $44(97 \%)$ patients after posterior capsulotomy (Fig. 3.). The improvement in mean Snellen visual acuity under glare conditions from 0.18 (SD 0.06) to 0.40 (SD 0.16), an increase of 150 (SD 127)\% after treatment was highly statistically significant $(\mathrm{p}<<0.001)$.

Standard Snellen visual acuity did not improve following capsulotomy in 16 (35\%) eyes. Of these 16 eyes, 14 showed an improvement in Snellen visual acuity under glare conditions. A statistically significant $(\mathrm{p}<0.001)$ increase in mean Snellen visual acuity under glare conditions from 0.21 (SD 0.07) to 0.37 (SD 0.18) was achieved in these eyes.

The difference between standard Snellen visual acuity and Snellen visual acuity under glare conditions in the number of patients showing an improvement following posterior capsulotomy (30 in the former versus 44 in the latter) was statistically significant $(\mathrm{p}<0.001)$.

\section{DISCUSSION}

This study confirms the clinical value of glare testing in evaluating pseudophakes with posterior capsule opacification who complain of visual disability in spite of good
Snellen visual acuity. Following posterior capsulotomy, Snellen visual acuity under glare conditions improved in significantly more patients than standard Snellen visual acuity. Glare testing was particularly useful in the subgroup of 16 eyes whose standard Snellen visual acuity did not improve after capsulotomy.

We cannot suggest a threshold below which Snellen visual acuity under glare conditions can serve as an indication for posterior capsulotomy. Complaints of functional visual disability in the presence of an opacified posterior capsule will continue to play an important role in deciding to perform posterior capsulotomy in pseudophakes. However, routine glare testing before and after posterior capsulotomy in pseudophakes with good Snellen visual acuity provides the ophthalmologist with objective evidence of visual improvement.

We thank Mrs. Anne Lewis for helping to organise the study and Mrs. M. Spink for secretarial assistance.

Key words: Glare test, Intraocular lens, Neodymium-YAG laser, Posterior capsule opacification, Posterior capsulotomy, Pseudophakia, Visual acuity, Visual disability.

\section{REFERENCES}

1. Masket S. Reversal of glare after cataract surgery. J Cataract Refract Surg 1989, 15: 165-8.

2. Nadler DJ, Jaffe NS, Clayman HM, Jaffe MS, Luscombe SM. Glare disability in eyes with intraocular lenses. Am J Ophthalmol 1984, 97: 43-7.

3. Terry AC, Stark WJ, Maumenee AE, Fagadau W. Neodymium-YAG laser for posterior capsulotomy. Am J Ophthalmol 1983, 96: 716-18.

4. Knighton RW, Slomovic AR, Parrish RK. Glare measurements before and after Neodymium-YAG laser posterior capsulotomy. Am J Ophthalmol 1985, 100: 708-13. 